\title{
Evaluation of Thiol/Disulfide Homeostasis in Bronchiectasis
}

\author{
Songul Ozyurt $\mathbb{D},{ }^{1}$ Neslihan Ozcelik $\mathbb{D},{ }^{1}$ Bilge Yilmaz Kara $\mathbb{D}^{1},{ }^{1}$ Medeni Arpa $\left(\mathbb{D},{ }^{2}\right.$ \\ Yavuz Metin $\left(\mathbb{D},{ }^{3}\right.$ Ozcan Erel $\mathbb{D}^{4},{ }^{4}$ and Salim Neselioglu $\mathbb{D i D}^{4}$ \\ ${ }^{1}$ Recep Tayyip Erdogan University, Training and Research Hospital, Chest Disease, Rize, Turkey \\ ${ }^{2}$ Recep Tayyip Erdogan University, Training and Research Hospital, Medical Biochemistry, Rize, Turkey \\ ${ }^{3}$ Ankara University, Medical Faculty Ibn i Sina Hospital, Department of Radiology, Ankara, Turkey \\ ${ }^{4}$ Yildirim Beyazit University, Faculty of Medicine, Department of Medical Biochemistry, Ankara, Turkey \\ Correspondence should be addressed to Neslihan Ozcelik; neslihan.ozcelik@erdogan.edu.tr
}

Received 16 September 2021; Revised 14 December 2021; Accepted 13 January 2022; Published 29 January 2022

Academic Editor: Youfeng Zhu

Copyright (C) 2022 Songul Ozyurt et al. This is an open access article distributed under the Creative Commons Attribution License, which permits unrestricted use, distribution, and reproduction in any medium, provided the original work is properly cited.

\begin{abstract}
Purpose. Thiols are sulfhydryl-containing organic compounds that have an important role in preventing cellular oxidative stress. This study compares the blood oxidative stress marker levels in bronchiectasis cases during their stable periods with healthy controls. Materials and Methods. Seventy-seven patients ( 49 patients with stable bronchiectasis/28 healthy controls), followed up by the chest disease clinic, were included in the study. Peripheral blood thiol-disulfide parameters (NT: native thiol (-SH); TT: total thiol (-SH + SS); SS: disulfide (-SS); SS-SH: disulfide/native thiol index; SS-TT: disulphide/total thiol index; SH-TT: native thiol/total thiol index), and ischemia-modified albumin (IMA) levels were examined in the stable bronchiectasis group and the control group. Thiol-disulfide homeostasis was evaluated using a novel and automated assay. Findings and Result. Blood native thiol levels in patients with stable bronchiectasis were found to be significantly higher compared with healthy controls. A positive correlation between the total airway disease score and IMA levels was present. Our findings revealed that native thiol levels, which constitute a part of the antioxidant defense system, are increased in patients with stable bronchiectasis.
\end{abstract}

\section{Introduction}

Bronchiectasis is a chronic airway disease characterized by abnormal degradation and widening of large airways, that is, bronchi and bronchioles. Etiologies of bronchiectasis include cystic fibrosis, primary ciliary dyskinesia, genetic anomalies, immune deficiency syndromes, modified host defense and autoimmune diseases, significant infections, and acquired disorders [1]. In bronchiectasis, airways show gradual damage due to proteinase enzymes secreted by epithelial cells, neutrophil-produced reactive oxygen radicals, eosinophils, macrophages, chronic microbial infection, airway inflammation, and interaction of substances harming the tissues [1].

It is produced due to the normal metabolism or various reasons such as ischemia-reperfusion, aging, radiation, high oxygen pressure, inflammation, and exposure to chemical agents, and oxygen free radicals [2] cause metabolic, structural, and functional damages, which may lead to cell death by reacting to various cellular components and macromolecules. An excessive number of free radicals produced during inflammation repress the antioxidant defense systems, which leads to considerable damage in the airway epithelial cells and other airway structures [3]. Oxidative stress occurs when the production of harmful oxidants exceeds the detoxification capacity of the antioxidant defense system in the body.

Various studies suggest that oxidative stress potentially contributes to the pathogenesis of many respiratory diseases, including cystic fibrosis, pulmonary tuberculosis, and acute respiratory distress syndrome [4]. Furthermore, it has been shown that pulmonary diseases with chronic airway inflammation, such as chronic obstructive pulmonary disease (COPD) and bronchial asthma, increase oxidative stress indicators in the plasma $[5,6]$. 
Thiol is an organic compound containing a sulfhydryl group that plays a critical role in preventing cellular oxidative stress. Cysteine, through its functional thiol group, plays an important role in the prevention of oxidative damage in the body [7].

1.1. Biological Significance of Thiols and Disulfides. Thiols and disulfides play an important role in the stabilization of protein structures and regulation of protein and enzyme functions, receptors, carriers, $\mathrm{Na}-\mathrm{K}$ channel, and transcription. The dynamic thiol-disulfide homeostasis (TDH) is critical for antioxidant defense, detoxification, apoptosis, regulation of enzyme activities, transcription, and cellular signal transduction mechanisms [7]. Abnormal TDH contributes to the pathogenesis of various diseases, such as diabetes mellitus, cardiovascular diseases, malignancy, rheumatoid arthritis, chronic renal failure, Parkinson's disease, Alzheimer's disease, multiple sclerosis, and liver diseases [7]. Measuring the TDH involves assessing the native thiol [-SH](NT), dynamic disulfide [-S-S-] (DS), and total thiol $[(-\mathrm{SH})+(-\mathrm{S}-\mathrm{S}-)](\mathrm{TT})$ levels and the dynamic "-$\mathrm{SH} /-\mathrm{S}-\mathrm{S}-”$ homeostasis [7].

1.2. Ischemia-Modified Albumin (IMA). Ischemia-modified albumin (IMA) is an albumin form that displays ischemic and oxidative damage and goes through structural changes. It is an indicator that might be used in many cases, such as strokes and cerebrovascular accidents, mesenteric ischemia, myocardial ischemia, and musculoskeletal ischemia [8].

This study provides an assessment of the thiol-disulfide homeostasis, an indicator of the activity of the antioxidant system, and IMA levels, indicating oxidative damage, in patients with stable bronchiectasis without acute inflammation symptoms.

\section{Materials and Methodology}

Forty-nine patients diagnosed with stable bronchiectasis without acute infection symptoms or findings were included in the study. The control group included 28 healthy individuals of the same age and sex. The age, sex, and highresolution computed tomography (HRCT) findings of all patients were recorded. Peripheral blood samples (total, $4 \mathrm{~mL}$ ) were collected from the antecubital vein in heparinized tubes and were stored at $2^{\circ} \mathrm{C}-4^{\circ} \mathrm{C}$ for measuring the thiol-disulfide hemostasis parameters. The blood was centrifuged at $1500 \mathrm{~g}$ for $10 \mathrm{~min}$ to separate the plasma. The separated plasma was then stored at $-80^{\circ} \mathrm{C}$ until analysis. The ethical committee of the Department of Medicine of Recep Tayyip Erdogan University approved the study (decision no. 2021/92).

2.1. Inclusion Criteria for the Study. The inclusion criteria are as follows:

(i) Being older than 18 years

(ii) Detection of bronchiectasis on the HRCT scan (iii) Being in a stable condition during the course of the disease (stability was defined as the absence of acute inflammation and antibiotic use within four weeks before the application; absence of acute inflammation was defined as the lack of the following symptoms: absence of an increase in dyspnea, cough and sputum volume, or purulence)

(iv) Having no smoking habits

(v) Not having chronic diseases such as hypertension, diabetes mellitus, chronic kidney disease, and coronary artery disease, malignancy, or rheumatological diseases

\subsection{Exclusion Criteria. The exclusion criteria are as follows:}

(i) Patients under 18 years of age

(ii) Patients with acute exacerbation of bronchiectasis (an increase in dyspnea, cough and sputum volume, or purulence)

(iii) Patients with an infectious or inflammatory condition (infections in other parts of the body, e.g., urinary tract infection and inflammatory arthritis)

2.3. Diagnosing and Scoring Bronchiectasis. Bronchiectasis was diagnosed using HRCT. Normally, airways are not visible in the lung parenchyma within $1 \mathrm{~cm}$ of the costal pleura. Furthermore, the inner diameter of the bronchi is equal to that of the accompanying pulmonary artery within the same bifurcation level. In adults, the ratio of the bronchoarterial diameter being lower than one is considered abnormal, and the visibility of the peripheral airways within $1 \mathrm{~cm}$ in the costal pleura is considered in favor of a bronchiectasis diagnosis [1]. The presence and prevalence of bronchiectasis were examined in accordance with the findings from the HRCT scans of the patients, and each lobe was evaluated individually based on the Bhalla scoring system [9]. Based on this classification, the bronchiectasis rate was scored as follows: 0: none; 1 : less than $25 \%$ of the lobe volume; 2 : between $25 \%$ and $50 \%$ of the lobe volume; and 3: more than $50 \%$ of the lobe volume. The total score for each patient was calculated as the bronchiectasis severity score. Furthermore, based on the findings from the HRCT scans, wall thickness was evaluated with the following scores: 0 : no thickening; 1 : thickening between $25 \%$ and $50 \%$; 2 : thickening of more than 50\%; and 3: completely obliterated. Furthermore, accompanying symptoms, such as peribronchial thickening, small airway pathologies, and mosaic perfusion in the neighboring lung, were also evaluated and recorded using HRCT.

\subsection{Thiol-Disulfide Homeostasis Parameters.} Thiol-disulfide homeostasis parameters were measured using a novel automatic and spectrophotometric method described by Erel and Neselioglu [10]. Free functional thiol groups $(-\mathrm{SH})$ were extricated by decreasing disulfide bonds (-S-S-) using sodium borohydride (NaBH4). Unused $\mathrm{NaBH} 4$ remnants were completely removed using 
formaldehyde. This prevented further reduction of $5,5^{\prime}$ dithiobis-2-nitrobenzoic acid (DTNB) as well as any disulfide bonds resulting from possible reactions with DTNB. Total thiol groups including reduced and native thiol groups were determined after reactions with DTNB. The disulfide parameter value can be calculated as half of the native thiol content and total thiol content. Disulfide/total thiol, disulfide/native thiol, and native thiol/total thiol ratios were calculated.

IMA levels were analyzed using the rapid and colorimetric method defined by Bar-Or et al. [11].

2.5. Statistical Analysis. SPSS 17.0 (Chicago Inc., 2008) and MedCalc ${ }^{\circledR}$ Statistical Software version 19.8 (MedCalc Software Ltd, Ostend, Belgium; https://www.medcalc.org; 2021) were used for the statistical analysis. Categorical variables were indicated as frequency $(n)$ and percentage (\%). The fitness of the continuous variables to normal distribution was tested using the Kolmogorov-Smirnov method. The NT and TT levels, identified to display a normal distribution, were indicated as mean and standard deviation, while other laboratory parameters (disulfide, SS-SH, SS-TT, SH-TT, and IMA) were indicated as median and interquartile range as they do not display a normal distribution. Student's $t$-test and Mann-Whitney $U$ tests were used for the binary comparison of the continuous variables. As for the categorical variables, they were analyzed using the Pearson chisquared and Fisher's exact tests. The correlation between bronchiectasis and oxidative stress parameters was analyzed using Spearman's correlation test. $p<0.05$ was accepted for significance.

\section{Findings}

The data for 77 participants ( 49 patients with stable bronchiectasis and 28 healthy controls) were compared. The mean age was 58.8 years; $51.9 \%$ of the participants $(n=40)$ were females, and $48.1 \%(n=37)$ were males. Both groups were found to be similar in terms of age and sex distribution $(p>0.05$; Table 1$)$.

The mean NT levels in the blood were found to be significantly higher in patients with stable bronchiectasis compared with healthy controls ( $p=0.047$; Figure 1$)$. Other $\mathrm{TDH}$ parameters (mean total thiol levels, median level distributions of disulfide, SS-SH, SS-TT, and SH-TT indices) and IMA were found to be similar for both groups $(p>0.05$; Table 1).

The examination of the prevalence of bronchiectasis revealed that the disease occurred bilaterally in multiple lobes in $53.1 \%$ of the patients, making it the most frequent incidence. Table 2 shows the other variables concerning the disease.

The correlation between the thiol-disulfide indices and IMA levels and bronchiectasis disease scores was also examined. A positive, medium-level, and statistically significant correlation was identified between the IMA levels in the blood and the total small airway disease scores $($ rho $=0.300$; $p=0.036)$. No significant correlation was found between the other parameters and the variables concerning bronchiectasis ( $p>0.05$ for all) (Table 3$)$. The correlation between the IMA levels in the blood and total small airway disease scores is shown in Figure $2(r=0.33, p=0.022)$.

\section{Discussion}

Our study reported higher NT levels in patients with stable bronchiectasis compared with healthy controls. Furthermore, a positive correlation between the total airway disease scores calculated for bronchiectasis and IMA levels was detected.

Reactive oxygen species (ROS), such as the hydroxyl radical, superoxide radical, and hydrogen peroxide, are produced during cellular metabolism. The insufficiency of antioxidants protecting the organism from the adverse effects of these substances disrupts the oxidative balance, causing oxidative stress. Elevated ROS levels in oxidative stress causes cell damage and cellular death by harming macromolecules within cells, such as lipids, proteins, and DNA [12]. As is the case in many diseases, oxidative stress plays an important role in the pathogenesis of various pulmonary diseases, such as COPD, asthma, pneumonia, tuberculosis, and bronchiectasis [13]. Previous studies reported significant increases in the oxidants in patients with lung diseases characterized by chronic inflammation such as bronchiectasis $[5,14,15]$.

As for thiols, they constitute an important component of the plasma antioxidant system, where they reverse the effects of the oxidants and protect the organism from their harmful effects [7]. The literature review did not reveal any studies assessing TDH in bronchiectasis. Although existing studies in the literature seem to concentrate on the increase in the oxidant capacity, there are also studies reporting an increase in antioxidant capacity against increased oxidation [16]. In their study conducted on pediatric patients with bronchiectasis, Gedik et al. demonstrated that chronic inflammation also increases the total antioxidant capacity (TAC) and total oxidant status (TOS) [3]. In a study conducted on patients with asthma, Cakmak et al. reported increases in both TAC and TOS levels [17]. Being in line with the results of these studies, we also reported higher native thiol levels in our patients with stable bronchiectasis compared with the healthy control group, which shows that the antioxidant system may be functional in patients with stable bronchiectasis.

Metals such as cobalt, copper, and nickel are connected to the last amino-terminal region in the albumin structure. In cases such as hypoxia, acidosis, and free-radical damage, the connection of these metals is diminished, which changes the albumin structure, producing "ischemia-modified albumin" (IMA) [18]. It was reported that, in many chronic inflammatory pulmonary diseases and hypoxic cases (pleural effusion, pulmonary embolism, etc.), serum IMA levels increase [19-24]. Furthermore, IMA was also reported to reflect disease severity. Our study examines IMA levels as one of the indicators of oxidative stress. When comparing the results with those of healthy controls, they seem to be similar. However, a positive correlation was spotted upon examining the relationship with the small airway disease score. 
TABLE 1: Comparison between the data of patients with stable bronchiectasis $(n=49)$ and the healthy control group $(n=28)$.

\begin{tabular}{|c|c|c|c|c|c|}
\hline & $\begin{array}{c}\text { Total } \\
n=77\end{array}$ & $\begin{array}{c}\text { Bronchiectasis } \\
n=49\end{array}$ & $\begin{array}{c}\text { Control } \\
n=28\end{array}$ & $\begin{array}{c}\text { Statistic } \\
\mathrm{T}, z \text {, or } \chi^{2}\end{array}$ & $p$ value \\
\hline Age (years) ${ }^{\mathrm{a}}$ & $58.8 \pm 13.9$ & $57.7 \pm 13.1$ & $60.6 \pm 15.4$ & -0.864 & 0.390 \\
\hline Sex, $n(\%)$ & & & & 0.067 & 0.796 \\
\hline Female & $40(51.9)$ & $26(53.1)$ & $14(50.0)$ & & \\
\hline Male & $37(48.1)$ & $23(46.9)$ & $14(50.0)$ & & \\
\hline \multicolumn{6}{|l|}{ Laboratory } \\
\hline Native thiol $(\mu \mathrm{mol} / \mathrm{L})^{\mathrm{a}}$ & $372.9 \pm 81.9$ & $386.8 \pm 83.0$ & $348.4 \pm 75.3$ & 2.017 & 0.047 \\
\hline Total thiol $(\mu \mathrm{mol} / \mathrm{L})^{\mathrm{a}}$ & $405.1 \pm 84.4$ & $418.6 \pm 85.9$ & $381.3 \pm 77.4$ & 1.900 & 0.061 \\
\hline Disulfide $(\mu \mathrm{mol} / \mathrm{L})^{\mathrm{b}}$ & $15.20 \pm 8.15$ & $14.50 \pm 9.07$ & $15.92 \pm 7.04$ & -0.699 & 0.485 \\
\hline SS-SH index $(\%)^{\mathrm{b}}$ & $4.013 \pm 2.18$ & $3.788 \pm 1.96$ & $4.489 \pm 2.05$ & -1.641 & 0.101 \\
\hline SS-TT index $(\%)^{\mathrm{b}}$ & $3.714 \pm 1.85$ & $3.521 \pm 1.68$ & $4.119 \pm 1.72$ & -1.641 & 0.101 \\
\hline SH-TT index $(\%)^{\mathrm{b}}$ & $92.57 \pm 3.70$ & $92.95 \pm 3.36$ & $91.76 \pm 3.44$ & -1.641 & 0.101 \\
\hline Classic IMA $(\mathrm{AU})^{\mathrm{b}}$ & $0.457 \pm 0.06$ & $0.457 \pm 0.05$ & $0.460 \pm 0.07$ & -0.752 & 0.452 \\
\hline
\end{tabular}

${ }^{a}$ Arithmetic mean (standard deviation). ${ }^{b}$ Median (interquartile range). ${ }^{*}$ Fisher's exact test. NT: native thiol (-SH); TT: total thiol (-SH + SS); SS: disulfide (-SS); IMA: ischemia-modified albumin; AU: absorbance unit; SS-SH: disulfide/native thiol index; SS-TT: disulfide/total thiol index; SH-TT: native thiol/total thiol index.

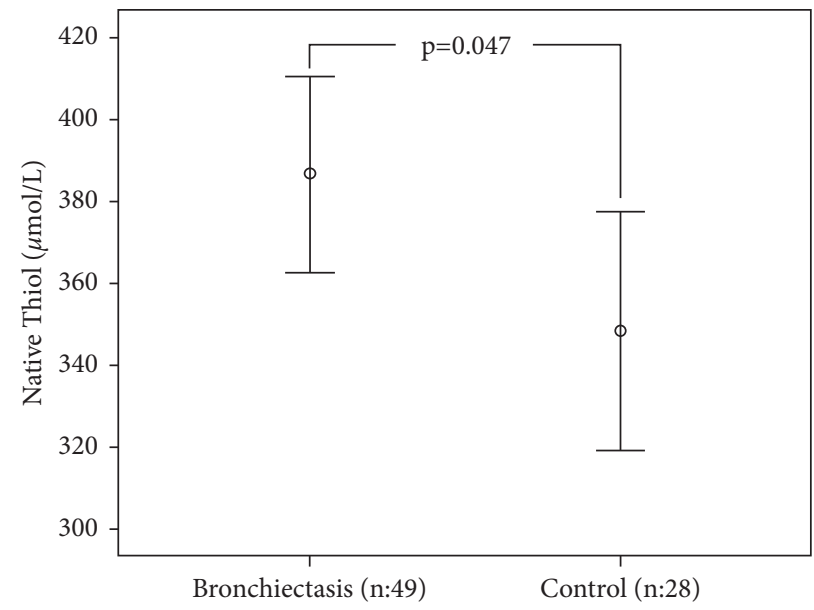

Figure 1: Comparison between the native thiol levels in patients with bronchiectasis and the control group with an error bar graph.

TABLE 2: Disease-related features of patients with stable bronchiectasis $(n=49)$.

\begin{tabular}{lc}
\hline & Results \\
\hline Bronchiectasis, $n(\%)$ & $5(10.2)$ \\
Right, single lobe & $3(6.1)$ \\
Left, single lobe & $7(14.3)$ \\
Bilateral, in one lobe each & $4(8.2)$ \\
Right, in multiple lobes & $4(8.2)$ \\
Left, in multiple lobes & $26(53.0)$ \\
Bilateral, in multiple lobes & $49(100.0)$ \\
Total & $5 \pm 4.5$ \\
Total BE score & $5.8 \pm 3.7$ \\
Total wall thickness (mm) & a \\
Total small airway disease score & $3 \pm 2.5$ \\
Total mosaic perfusion & \\
\hline
\end{tabular}

${ }^{\mathrm{a}}$ Arithmetic mean (standard deviation). ${ }^{\mathrm{b}}$ Median (interquartile range).

Inflammation is expected to be more intense during the acute inflammation period. The lack of differences between the IMA levels in our patients and healthy controls may be attributed to the stability of our patients. Alternatively, the positive correlation with the calculated total small airway disease score indicates a potential relationship with disease severity.
4.1. Limitations of the Study and Suggestions for Future Studies. The basic limitations of our study include its monocentral nature and the small number of patients. The study was conducted on patients with stable bronchiectasis. Since patients in the acute exacerbation period were excluded, the study excludes any results regarding the state of 
TABLE 3: Analyzing the correlation between the oxidative stress parameters in the bronchiectasis group $(n=49)$ with disease scores.

\begin{tabular}{lccccc}
\hline & & $\begin{array}{c}\text { Total bronchiectasis } \\
\text { score }\end{array}$ & $\begin{array}{c}\text { Total wall } \\
\text { thickness }\end{array}$ & $\begin{array}{c}\text { Total small airway disease } \\
\text { score }\end{array}$ & $\begin{array}{c}\text { Total mosaic perfusion } \\
\text { score }\end{array}$ \\
\hline \multirow{2}{*}{ Native thiol (-SH) } & Rho & 0.01 & 0.01 & -0.08 & -0.03 \\
Total thiol & $p$ value & 0.96 & 0.93 & 0.59 & 0.83 \\
$(-S H+$ SS) & Rho & 0.03 & 0.01 & -0.05 & -0.01 \\
Disulfide (-SS) & $p$ value & 0.83 & 0.96 & 0.72 & 0.95 \\
& Rho & 0.12 & 0.09 & 0.12 & 0.15 \\
SS-SH index & value & 0.34 & 0.53 & 0.43 & 0.30 \\
& Rho & 0.17 & 0.13 & 0.16 & 0.18 \\
SS-TT index & value & 0.24 & 0.39 & 0.26 & 0.21 \\
& Rho & 0.17 & 0.13 & 0.16 & 0.18 \\
SH-TT index & $p$ value & 0.24 & 0.39 & -0.16 & 0.21 \\
Rho & $p$ value & -0.17 & -0.13 & 0.26 & -0.18 \\
Classic IMA & Rho & 0.24 & 0.39 & $\mathbf{0 . 3 0}$ & 0.21 \\
& $p$ value & 0.95 & -0.08 & $\mathbf{0 . 0 4}$ & .25 \\
\hline
\end{tabular}

IMA: ischemia-modified albumin; SS-SH: disulfide/native thiol index; SS-TT: disulfide/total thiol index; SH-TT: native thiol/total thiol index. * ${ }^{*}$ correlation is significant at the 0.05 level (2-sided).

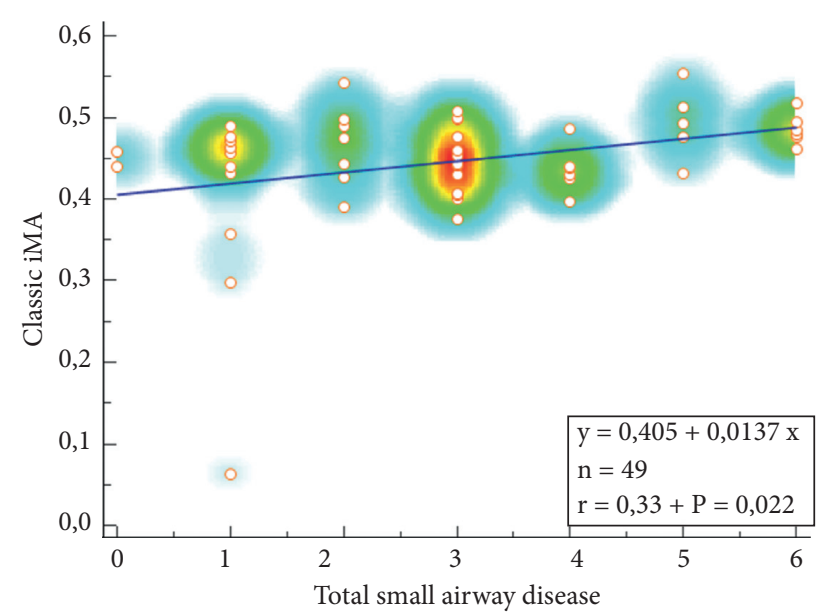

FIGURE 2: The correlation between the IMA levels in the blood and total small airway disease scores.

the antioxidant system in these patients. Further studies including both types of patients are required.

\section{Conclusions}

Our study demonstrated that, in bronchiectasis, thiol levels were increased, which indicated that the antioxidant system is active. It should be noted that, in bronchiectasis, substantial evidence is emerging suggesting persistent chronic inflammation in the stable period as well.

Considering our study and previous studies, the need for approaches supporting the antioxidant system in addition to treatments targeting infections becomes evident.

\section{Data Availability}

The data that support the findings of this study are available from the corresponding author upon reasonable request.

\section{Conflicts of Interest}

The authors declare no conflicts of interest.

\section{References}

[1] A. T Hill, A. L Sullivan, J. D Chalmers et al., "British Thoracic Society Guideline for bronchiectasis in adults," Thorax, vol. 74, no. Suppl 1, pp. 1-69, 2019.

[2] B. S. Berlett and E. R. Stadtman, "Protein oxidation in aging, disease, and oxidative stress," Journal of Biological Chemistry, vol. 272, no. 33, pp. 20313-20316, 1997.

[3] A. H. Gedik, E. Çakir, A. Vehapoğlu Türkmen, ÖF. Özer, and S. B. Kaygusuz, "Total oxidant and antioxidant status and paraoxonase 1 levels of children with noncystic fibrosis bronchiectasis," Turkish Journal of Medical Sciences, vol. 50, pp. 1-7, 2020.

[4] W. MacNee, "Oxidative stress and lung inflammation in airways disease," European Journal of Pharmacology, vol. 429, no. 1-3, pp. 195-207, 2001.

[5] R. R. Rai and M. S. Phadke, "Plasma oxidant-antioxidant status in different respiratory disorders," Indian Journal of Clinical Biochemistry, vol. 21, no. 2, pp. 161-164, 2006.

[6] C. Cristóvão, L. Cristóvão, F. Nogueira, and M. Bicho, "Evaluation of the oxidant and antioxidant balance in the pathogenesis of chronic obstructive pulmonary disease," Revista portuguesa de pneumologia, vol. 19, pp. 70-75, 2013. 
[7] Ö. Erel and S. Erdoğan, "Thiol-disulfide homeostasis: an integrated approach with biochemical and clinical aspects," Turkish Journal of Medical Sciences, vol. 50, no. 7, pp. 1728-1738, 2020.

[8] D. C. Gaze, "Ischemia modified albumin: a novel biomarker for the detection of cardiac ischemia," Drug Metabolism and Pharmacokinetics, vol. 24, no. 4, pp. 333-341, 2009.

[9] M. Bhalla, N. Turcios, V. Aponte et al., "Cystic fibrosis: scoring system with thin-section CT," Radiology, vol. 179, no. 3, pp. 783-788, 1991.

[10] O. Erel and S. Neselioglu, "A novel and automated assay for thiol/disulphide homeostasis," Clinical Biochemistry, vol. 47, no. 18, pp. 326-332, 2014.

[11] D. Bar-Or, E. Lau, and J. V. Winkler, "A novel assay for cobalt-albumin binding and its potential as a marker for myocardial ischemia-a preliminary report," The Journal of Emergency Medicine, vol. 19, pp. 311-315, 2000.

[12] O. Özcan, H. Erdal, G. Çakırca, and Z. Yönden, "Oxidative stress and its impacts on intracellular lipids, proteins and DNA," J Clin Exp Invest, vol. 6, pp. 331-336, 2015.

[13] E. Bargagli, C. Olivieri, D. Bennett, A. Prasse, J. MullerQuernheim, and P. Rottoli, "Oxidative stress in the pathogenesis of diffuse lung diseases: a review," Respiratory Medicine, vol. 103, no. 9, pp. 1245-1256, 2009.

[14] A. van der Vliet, Y. M. W. Janssen-Heininger, and V. Anathy, "Oxidative stress in chronic lung disease: from mitochondrial dysfunction to dysregulated redox signaling," Molecular Aspects of Medicine, vol. 63, pp. 59-69, 2018.

[15] G. Olveira, C. Olveira, A. Dorado et al., "Cellular and plasma oxidative stress biomarkers are raised in adults with bronchiectasis," Clinical Nutrition, vol. 32, no. 1, pp. 112-117, 2013.

[16] A. Nadeem, S. K. Chhabra, A. Masood, and H. G. Raj, "Increased oxidative stress and altered levels of antioxidants in asthma," Journal of Allergy and Clinical Immunology, vol. 111, no. 1, pp. 72-78, 2003.

[17] A. Cakmak, D. Zeyrek, A. Atas, H. Celik, N. Aksoy, and O. Erel, "Serum prolidase activity and oxidative status in patients with bronchial asthma," Journal of Clinical Laboratory Analysis, vol. 23, no. 2, pp. 132-138, 2009.

[18] E. Sbarouni, P. Georgiadou, and V. Voudris, "Ischemia modified albumin changes-review and clinical implications," Clinical Chemistry and Laboratory Medicine (CCLM), vol. 49, pp. 177-184, 2011.

[19] S. Kimura, H. Yamaguchi, Y. Shikama et al., "Serum ischemiamodified albumin concentration may reflect long-term hypoxia in chronic respiratory disease: a pilot study," Clinical Chemistry and Laboratory Medicine (CCLM), vol. 56, no. 12, pp. e288-e290, 2018.

[20] K.-Y. Yang and V. Y.-F. Su, Serum Oxidative Stress and Chronic Obstructive Pulmonary Disease, LWW, 2015.

[21] M. Doğru, H. Akoğlu, M. F. Kılınçkaya, and G. Ülfer, Ischemia-modified Albumin Levels in Children with Asthma: A Pilot Study, 2018.

[22] S. Turedi, A. Gunduz, A. Mentese et al., "Value of ischemiamodified albumin in the diagnosis of pulmonary embolism," The American Journal of Emergency Medicine, vol. 25, no. 7, pp. 770-773, 2007.

[23] O. Dikensoy, N. Celik, S. Kul, B. Gogebakan, H. Bayram, and R. W. Light, "Ischemia modified albumin in the differential diagnosis of pleural effusions," Respiratory Medicine, vol. 105, no. 11, pp. 1712-1717, 2011.

[24] M. Bolatkale, M. Duger, G. Ülfer et al., "A novel biochemical marker for community-acquired pneumonia: ischemiamodified albumin," The American Journal of Emergency Medicine, vol. 35, no. 8, pp. 1121-1125, 2017. 